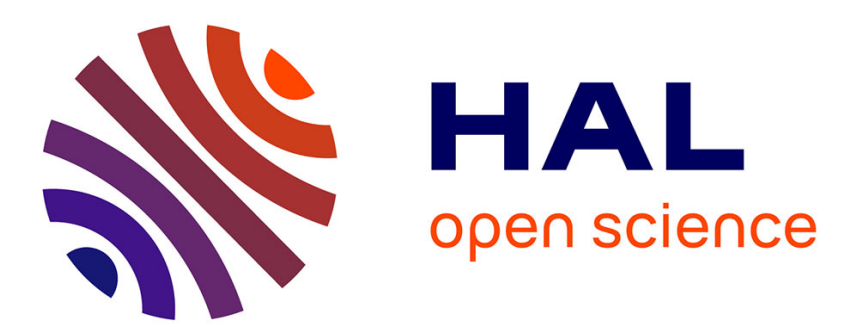

\title{
A Thin Film Fluid Structure Interaction Model for the Study of Flexible Structure Dynamics in Centrifugal Pumps
}

\author{
Abdulaleem Albadawi, Mathieu Specklin, R. Connolly, Yan Delauré
}

\section{To cite this version:}

Abdulaleem Albadawi, Mathieu Specklin, R. Connolly, Yan Delauré. A Thin Film Fluid Structure Interaction Model for the Study of Flexible Structure Dynamics in Centrifugal Pumps. Journal of Fluids Engineering, 2018, 141 (6), pp.500 - 600. 10.1115/1.4041759 . hal-02472899

\section{HAL Id: hal-02472899 \\ https://hal.science/hal-02472899}

Submitted on 10 Feb 2020

HAL is a multi-disciplinary open access archive for the deposit and dissemination of scientific research documents, whether they are published or not. The documents may come from teaching and research institutions in France or abroad, or from public or private research centers.
L'archive ouverte pluridisciplinaire HAL, est destinée au dépôt et à la diffusion de documents scientifiques de niveau recherche, publiés ou non, émanant des établissements d'enseignement et de recherche français ou étrangers, des laboratoires publics ou privés. 


\section{A. Albadawi \\ School of Mechanical and \\ Manufacturing Engineering, \\ Dublin City University, \\ Glasnevin, \\ Dublin D9, Ireland \\ e-mail: abdulaleem.albadawi@sulzer.com \\ M. Specklin \\ Sulzer Pump Solutions Ireland Ltd. \\ School of Mechanical and \\ Manufacturing Engineering, \\ Dublin City University, \\ A Thin Film Fluid Structure Interaction Model for the Study of Flexible Structure Dynamics in Centrifugal Pumps}

Glasnevin,

Dublin D9, Ireland

e-mail: mathieu.specklin2@mail.dcu.ie

\section{R. Connolly \\ Global Technology, \\ Pumps Equipment, \\ Sulzer Pump Solutions Ireland Ltd. \\ Clonard Road, \\ Wexford Y35 YE24, Ireland \\ e-mail: abdulaleem.albadawi@sulzer.com}

\author{
Y. Delauré \\ School of Mechanical and \\ Manufacturing Engineering \\ Dublin City University, \\ Glasnevin, \\ Dublin D9, Ireland \\ e-mail: yan.delaure@dcu.ie
}

\begin{abstract}
This paper describes a fluid-structure interaction (FSI) model for the study of flexible cloth-like structures or the so-called rags in flows through centrifugal pumps. The structural model and its coupling to the flow solver are based on a Lagrangian formulation combining structural deformation and motion modeling coupled to a sharp interface immersed boundary model (IBM). The solution has been implemented in the open-source library OpenFOAM relying in particular on its PIMPLE segregated Navier-Stokes pressure-velocity coupling and its detached eddy simulation (DES) turbulence model. The FSI solver is assessed in terms of its capability to generate consistent deformations and transport of the immersed flexible structures. Two benchmark cases are covered and both involve experimental validation with three-dimensional (3D) structural deformations of the rag captured using a digital image correlation (DIC) technique. Simulations of a rag transported in a centrifugal pump confirm the suitability of the model to inform on the dynamic behavior of immersed structures under practical engineering conditions.
\end{abstract}

\section{Introduction}

The focus of the present fluid structure interaction (FSI) study is the transport of immersed structures in centrifugal pumps designed for waste water systems. These pumps typically need to handle particle-laden flow but also thin flexible cloth-like structures or the so-called rags. Their accumulation within the volute affects hydraulic efficiency but can also quickly lead to a full pump blockage. The issue can cause severe disruption to sewage distribution networks and has motivated continued research in hydraulic design. Pump optimization for this type of application often involves some form of compromise between hydraulic efficiency and anticlogging performances. A few studies [1-5] have attempted to characterize and quantify experimentally the mechanisms that lead to blockage and their impact on pump hydraulic performance. It has proven very difficult, however, to understand the behavior of thin flexible structures, so that the effectiveness and consequences of more subtle pump design changes are difficult to assess. The aim of this study is to propose and validate a computational model capable of capturing both solid and liquid phases to characterize their interaction with the pump impeller. There is very limited evidence of published research having been carried out on such computational modeling to date. The more recent work by Jensen et al. [6,7] for the study of clogging in waste water pumps based on a discrete element method is one notable exception to this. In this method, interparticles forces approximate the real strain-stress characteristics of the rag. Computational fluid dynamics simulations of centrifugal pumps were performed in Refs. [8,9] and [10,11] where FSI modeling has been used to study the impeller vibrations induced by nonsymmetrical hydrodynamic loads. The Design of Experiments study of a single blade impeller pump of Ref. [12], the Constant-Velocity volute analysis of Ref. [13], and the validation of computational fluid dynamics simulations of Ref. [14] are to be noted for the development and design of pump impellers and volutes. However, none of these investigations consider the transport of secondary flexible objects through the pump.

The present study relies on a model which couples the sharp interface immersed boundary method (IBM) of Specklin and Delauré [15] to capture moving rigid boundaries with the diffuse immersed boundary (IB) FSI model of Refs. [16-20] for immersed flexible structures. The FSI and collision models have previously been partially validated by considering two sets of well-documented experimental benchmark cases characterized by highly dynamic response of the flexible structure where oscillations are promoted by the choice of bending and torsion rigidity for the material [21]. The first set concerns one-dimensional filaments oscillating under the effect of gravity with and without interaction with external flow [16]. The second involves the sustained flapping of a two-dimensional thin flexible membrane in external crossflow and has been extensively used in the literature [17,22-24]. This analysis confirmed that predictions of the structures' oscillations are in good agreement with experimental and numerical data available from the literature, and the reader is referred to Ref. [21] for a detailed assessment. Two new validation cases are proposed in the present article. Both rely on the experimental characterization of three-dimensional (3D) structural deformations for comparison with model predictions and consider two types of boundary conditions for the immersed structure (free and imposed rigid body motion with two-way coupling). The aim is to assess the model's behavior and accuracy with material properties that are more representative of those tested with real pumps. 
The use of IBM to capture the pump's impeller is justified by reference to the pump performance curves. The models are then adapted to simulate the interaction of an immersed rag with the pump volute and the rotating impeller at two different flow rates.

The paper is divided into four sections in addition to the introduction. Section 2 describes the formulation of the problem. The model is then assessed in Sec. 3 for both flag oscillations and rag transport in freestreams. This analysis relies on experimental measurements of three-dimensional deformations of the immersed structure obtained with a digital image correlation (DIC) technique. A brief description of the experimental system is also provided in this section. Finally, the simulation of flow in centrifugal pumps with and without FSIis discussed in Sec. 4 in part to describe the processes leading to clogging of centrifugal pump. The conclusions and findings of the paper are presented in Sec. 5.

\section{Theoretical Background}

2.1 Governing Equations. The model solves the threedimensional incompressible Navier-Stokes equations on an Eulerian mesh (Eqs. (2) and (1)) for the flow velocity $\mathbf{u}$ and pressure $p$ and the structure motion equation (Eq. (3)) on a Lagrangian twodimensional mesh (with curvilinear coordinates $\left(s_{1}, s_{2}\right)$ ) to update the Lagrangian point positions $\mathbf{X}$

$$
\begin{gathered}
\rho_{f}\left(\frac{\partial \mathbf{u}}{\partial t}+\mathbf{u} \cdot \nabla \mathbf{u}\right)=-\nabla p+\mu \nabla^{2} \mathbf{u}+\mathbf{f}_{\mathrm{ibm}}+\mathbf{f}_{\mathrm{fsi}} \\
\nabla \cdot \mathbf{u}=0 \\
\left.\rho_{1} \frac{\partial^{2} \mathbf{X}}{\partial t^{2}}=\sum_{i, j=1}^{2}\left[\frac{\partial}{\partial s_{i}}\left(\sigma_{i j} \frac{\partial \boldsymbol{X}}{\partial s_{j}}\right)-\frac{\partial^{2}}{\partial s_{i} \partial s_{j}} \quad \gamma_{i j} \frac{\partial^{2} \mathbf{X}}{\partial s_{i} \partial s_{j}}\right)\right]+\rho_{1} \mathbf{g}-\mathbf{F}_{\mathrm{fsi}} \\
+\mathbf{F}_{c}
\end{gathered}
$$

where $\rho_{f}$ and $\mu$ are the fluid density and the dynamic viscosity, respectively, and $\rho_{1}=\rho_{s}-\rho_{f} c$ is the difference between the solid mass per unit surface area $\rho_{s}$ and the fluid density multiplied by the thickness of the flexible structure $c$. Neutrally buoyant cases $\left(\rho_{1}=0\right)$ are not considered here and a gravity term is included $\left(\rho_{1} \mathbf{g}\right)$. The sharp interface IBM method of Specklin and Delauré [15] is adopted to account for the effect of immersed surfaces from rigid bodies. It defines the momentum source $\mathbf{f}_{\mathrm{ibm}}$ in Eq. (1) to correct the pressure and velocity boundary conditions taking account of the position and orientation of the immersed surface. The motion equation determines the acceleration of Lagrangian points in response to internal structural stresses and interactions of the immersed flexible object with the fluid $\left(\mathbf{F}_{\mathrm{fsi}}\right)$ and all rigid boundaries through a collision term $\left(\mathbf{F}_{c}\right)$. Internal stresses include tension and bending with the terms $\sigma_{i j}=$ $\varphi_{i j}\left(T_{i j}-T_{i j}^{0}\right)$ and $\gamma_{i j}=\zeta_{i j}\left(B_{i j}-B_{i j}^{0}\right)$, respectively. The variable $T_{i j}=\left(\partial X / \partial s_{i}\right) \cdot\left(\partial X / \partial s_{j}\right)$ models stretching when $i=j$ and shearing when $j \neq j$, while $B_{i j}=\left(\partial^{2} X / \partial s_{i} \partial s_{j}\right) \cdot\left(\partial^{2} X / \partial s_{i} \partial s_{j}\right)$ represents bending when $i=j$ and twisting when $j \neq j$. The rigidity to tension and bending are determined by the proportionality constants $\varphi_{i j}$ and $\zeta_{i j}$, respectively. The superscript 0 denotes the initial time.

The test cases studied rely on three types of boundary conditions on Lagrangian points which are the (i) fixed condition ( $X=$ constant, $\partial^{2} X / \partial s_{i}^{2}=(0,0)$ for $i=1$ or 2$)$, (ii) clamped condition ( $X=$ constant, $\partial X / \partial s_{i}=$ constant for $i=1$ or 2 ), and (iii) free end condition $\partial^{2} X / \partial s_{i}^{2}=(0,0), \partial^{3} X / \partial s_{i}^{3}=(0,0)$ and $\sigma_{i j}=0, \gamma_{i j}=0$ for $i, j=1$ or 2 .

2.2 Discretization Technique and Solution Solver. The fluid flow is solved using the open source library OpenFOAM2.3.1 [25] and its PIMPLE pressure-velocity coupling method which is an iterative implementation of the pressure-implicit with splitting of operators (PISO) scheme [26]. The Euler implicit scheme is used for time advancement. The Gauss' theorem is applied to express the divergence terms as flux summations over the bounding cell faces. Momentum fluxes are treated using OpenFOAM's linear-upwind stabilized transport scheme defined as a blend of the unbounded second-order central differencing scheme weighted at 0.75 and a second-order central difference bounded scheme based on the normalized variable diagram [27]. The scheme has been shown to stabilize grid-sensitive spurious oscillations associated with the Central Differencing scheme. In a comparison with Bounded and Filtered Central Differencing scheme, it was found to be the most effective at suppressing pressure oscillations in scale-resolved large eddy simulations (LES) of subcritical external slightly compressible flow past a cylinder [28]. It should be noted that the improved stability is achieved at the cost of increased numerical dissipation as discussed by Krastev and Bella in their analysis of detached eddy simulation (DES) wall bounded flow simulations [29]. All other convective fluxes (e.g., $\nu_{t}$ and $\tilde{\nu}$ ) are interpolated using the bounded central scheme without blending. The Laplacian terms are again treated using the Gauss' theorem to express the volume integral as a sum of diffusive fluxes approximated in this case using a central differencing scheme. The second-order approximation is achieved, in this case, by using a deferred correction to account for nonorthogonal components.

The motion equation for the flexible immersed object is discretized following the finite difference approach introduced by Ref. [18]. Numerical tests indicated that stable solution can be achieved with the following time-step constraint: $\Delta s / \Delta t \geq 100$, where $\Delta s$ and $\Delta t$ are the Lagrangian space step size and the timestep size, respectively. The following discretized equation is solved on a staggered and uniform initial grid where bending coefficients are defined at the Lagrangian points, while the tension coefficients are defined at the midpoint between the Lagrangian points

$$
\begin{aligned}
{\left[\rho_{1} I-\Delta t^{2} K+\Delta t \frac{\lambda}{2}\right] \mathbf{X}^{n+1}=} & \left(2 \rho_{1}\right) \mathbf{X}^{n}-\left(\rho_{1}-\Delta t \frac{\lambda}{2}\right) \mathbf{X}^{n-1} \\
& +\Delta t^{2} \rho \mathbf{g}-\Delta t^{2} \mathbf{F}_{\mathrm{fsi}}^{n}+\Delta t^{2} \mathbf{F}_{C}^{n}
\end{aligned}
$$

2.3 Fluid Structure Interaction Coupling. The coupling between the flow and structural solvers is defined by $\mathbf{f}_{\text {fsi }}$ and $\mathbf{F}_{\text {fsi }}$ following the diffuse IBM of Refs. [17] and [18] as detailed in Ref. [21] and summarized below:

- Update the position of internal immersed rigid surfaces and associated Lagrangian points $(\mathbf{X})$ before calculating the IBM force $\mathbf{f}_{\text {ibm }}$ from Eq. (9).

- The forcing term, $\mathbf{F}_{\mathrm{fsi}}$, in the Lagrangian motion equation (Eq. (4)) is defined implicitly using two sets of Lagrangian points: the immersed boundary points $\left(\mathbf{X}_{\mathrm{ib}}\right)$ calculated directly from the local fluid velocity $\mathbf{U}_{\mathrm{ib}}$ and the structure points $(\mathbf{X})$ obtained from the solution of the flag motion equation

$$
\mathbf{F}_{\mathrm{fsi}}=-K_{\mathrm{fsi}}\left(\mathbf{X}_{\mathrm{ib}}^{n+1}-2 \mathbf{X}^{n}+\mathbf{X}^{n-1}\right)
$$

where $X_{\mathrm{ib}}^{n+1}=X_{\mathrm{ib}}^{n}+U_{\mathrm{ib}}^{n} \Delta t$ is the new estimated position of the Lagrangian points. The velocity $U_{\mathrm{ib}}^{n}$ at the position $X_{\mathrm{ib}}^{n}$ is obtained using linear interpolation from the velocity value at the closest cell center on the Eulerian frame. The superscript $n$ represents the time step. The term $K_{\mathrm{fsi}}$ is a large penalization constant [18].

- The Lagrangian forcing $\mathbf{F}_{\mathrm{fsi}}$ is transferred to the Eulerian grid, using a smoothed three-dimensional Dirac function, to define $\mathbf{f}_{\mathrm{fsi}}$ in Eq. (1)

$$
\delta(\mathbf{X})=\frac{1}{h^{3}} \varphi\left(\frac{x}{h}\right) \varphi\left(\frac{y}{h}\right) \varphi\left(\frac{z}{h}\right)
$$




$$
\varphi(r)= \begin{cases}\frac{1}{8}\left(3-2|r|+\sqrt{1+4|r|-4\left|r^{2}\right|}\right) & 0 \leq|r|<1 \\ \frac{1}{8}\left(5-2|r|+\sqrt{-7+12|r|-4\left|r^{2}\right|}\right) & 1 \leq|r|<2 \\ 0 & 2 \leq|r|\end{cases}
$$

where $h$ is the Eulerian mesh size. With this diffuse IBM, the Eulerian momentum source is influenced by all Lagrangian points which satisfy simultaneously $x / h<2, y / h<2$, and $z / h<2$. The source is determined from a surface integral over the Immersed surface $\Gamma$

$$
\mathbf{f}_{\mathrm{fsi}}^{n}=\int_{\Gamma} \mathbf{F}_{\mathrm{fsi}}^{n}(\Gamma, t) \delta\left(\mathbf{x}-\mathbf{X}^{n}(\Gamma, t)\right) d \Gamma
$$

where $\mathbf{x}$ is the position of the Eulerian cell center.

- The updated pressure and velocity fields at step $n+1$ are obtained from the solution of the transient Navier-Stokes equation using the PIMPLE pressure velocity coupling.

- The motion equation (Eq. (4)) is solved for the solid positions at time $n+1$ giving $\mathbf{X}^{n+1}$.

- Correct the Lagrangian point positions $(\mathbf{X})$ to account for collision using Eq. (11).

- Iterate through time.

2.4 Sharp Interface Immersed Boundary Model for Immersed Rigid Surfaces. The sharp immersed boundary method from Ref. [15] has been integrated with the diffuse IBM to also model static and moving surfaces from rigid immersed objects. The method is based on a penalization approach. The IBM penalization constant $K_{\mathrm{ibm}}$ represents the permeability of the immersed object and must be set to a very small value to model solids $\left(K_{\mathrm{ibm}} \ll 1\right)$. The immersed boundary force in Eq. (1) is written as

$$
\mathbf{f}_{\mathrm{ibm}}=\chi \frac{\mu_{\mathbf{f}}}{K_{\mathrm{ibm}}}\left(\mathbf{u}-\mathbf{u}_{\mathrm{rib}}\right)
$$

where the vector $\mathbf{u}_{\text {rib }}$ is the prescribed velocity of rigid immersed boundary. The constant $\chi$ is a characteristic function defined as

$$
\chi(x, t)= \begin{cases}1 & \text { if } x \text { is inside rigid domain } \\ 0 & \text { if } x \text { is inside fluid domain }\end{cases}
$$

In all cases considered in this paper, the penalization coefficient $K_{\mathrm{ibm}}$ is defined implicitly using a single constant penalization $K_{\mathrm{ibm}}=1 \times 10^{-6}$

2.5 Collision Model. Collision between flexible immersed structures and rigid boundaries (whether they are modeled by the sharp IBM method or as a wall boundary) is handled by maintaining a liquid film between the two surfaces. The position of the Lagrangian points (flexible object) is corrected if the minimum distance $\epsilon_{c}$ between the flexible object and the closest rigid boundary is less than a specific threshold $d_{t}$. The following correction is applied only for the Lagrangian points which satisfy this threshold:

$$
\mathbf{X}_{c}^{n+1}=\mathbf{X}^{n+1}+\mathbf{n}_{w}\left(d_{t}-\epsilon_{c}\right)
$$

where $\mathbf{n}_{w}$ is the unit normal to the rigid surface and $\mathbf{X}_{c}^{n+1}$ is the position of the Lagrangian point after correction.

Self-collision arising from the folding of the rag has not been observed in any of the simulations performed. Lower structural rigidity, stronger flow mixing, and larger flexible structures may combine to create conditions which are favorable to more complex structural deformation including the formation of selfintersecting manifolds. Although this would not be strictly incompatible with the Lagrangian model, it is likely to create sharp gradients and generate unstable conditions. It would be advisable in this case to adapt the collision model to include self-collision. The same general approach can be used. In this case, however, the corrective displacement would have to be spread between colliding Lagrangian points.

2.6 Turbulence Modeling. A DES model is coupled to the present Immersed Boundary Method, as described in Ref. [15] to model turbulence. The model implemented is the Spalart-Allmaras (SA) DES model of Ref. [30] which relies Reynolds-averaged Navier-Stokes modeling in the vicinity of wall boundaries but provides LES modeling capability in the rest of the flow domain. This is achieved by substituting $\tilde{d}=d-f_{d} \max \left(0, l_{\mathrm{SA}}-l_{\text {des }}\right)$ for the Reynolds-averaged Navier-Stokes length scale $l_{\mathrm{SA}}=d$ which is based, in the SA model, on the wall distance $d$ alone. $l_{\text {des }}=c_{\text {des }} \Delta$ is the LES length scale defined by $\Delta=(\Delta x \Delta y \Delta z)^{1 / 3}$. The turbulent inlet boundary condition is specified in terms of the modified turbulent viscosity with $\tilde{\nu} / \nu=3$. It should be noted that immersed rigid surfaces (pump impeller or immersed obstacles) and outer rigid (wall) boundaries are treated differently. The latter is handled using a boundary formulation. For turbulent flow, this involves imposing a Dirichlet condition for the modified viscosity $\tilde{\nu}=0$. In addition, Spalding's law of the wall was included to model momentum transfer at rigid wall boundaries [31]. For immersed rigid surfaces, the same condition for $\tilde{\nu}$ is imposed using in this case the sharp IBM reconstruction detailed in Ref. [15]. No turbulence correction has been specified for immersed flexible structures due to the diffuse nature of the IB method.

\section{Benchmark Cases}

The test cases considered here were selected to complement the previous validation detailed in Ref. [21] by covering material properties and flow conditions that are more relevant to the case of flow in centrifugal pumps. These conditions will be determined in terms of the Reynolds number $\operatorname{Re}=\rho_{f} U L / \mu$, the nondimensional bending rigidity $K_{B}=\zeta / \rho_{1} U^{2} L^{2}$, the nondimensional tension coefficient $K_{T}=\varphi / \rho_{1} U^{2}$, and the nondimensional mass ratio $\rho=\rho_{1} / \rho_{f} L$, where $L$ is the flag length and $U$ is the flow mean velocity. In all cases considered here, the gravitational acceleration is applied along the vertical direction and its magnitude is taken as $9.81 \mathrm{~m}^{2} / \mathrm{s}$.

3.1 Pendulum Oscillation in Air. The FSI model is validated here against experimental results for oscillations in air. The thin flexible structure is a paper sheet of know mechanical properties. It is clamped at the bottom of a $10 \mathrm{~cm}$ long bar connected by a straight arm to a horizontal axis with a bearing to minimize friction. The paper sheet is initially flat and is released from an initial inclination of $30 \mathrm{deg}$. After the release, the bar oscillates freely under the action of gravity and the only source of damping is assumed to be due to drag induced by FSI between the paper sheet and surrounding air. A sketch illustrating the experimental setup is shown in Fig. 1. The main purpose of these experimental measurements is to provide a qualitative assessment of the model prediction with structural constants determined from the first principle. The tension and bending coefficients can be obtained from the Young's modulus $E$, the thickness $\epsilon$, and the Poisson's ratio $\nu$ of the rag, as $\varphi=E \epsilon$ and $\zeta=E \epsilon^{3} / 12\left(1-\nu^{2}\right)$. The rectangular flag is cut from an $\mathrm{A} 4$ sheet of paper with the dimensions $(0.1 \mathrm{~m} \times 0.05 \mathrm{~m})$. The flag has a thickness of $100 \mu \mathrm{m}$ and a surface density of $80 \mathrm{~g} / \mathrm{m}^{2}$. The carrier phase is air (with density $1.25 \mathrm{~kg} / \mathrm{m}^{3}$ and dynamic viscosity of $2 \times 10^{-5} \mathrm{~m}^{2} / \mathrm{s}$ ). The properties of the paper are provided in Table 1 . The bending rigidity considered for this study is in agreement with values reported in the literature which varies from $1 \times 10^{-4} \mathrm{~N} \cdot \mathrm{m}$ for a paper of $80 \mathrm{~g} /$ 


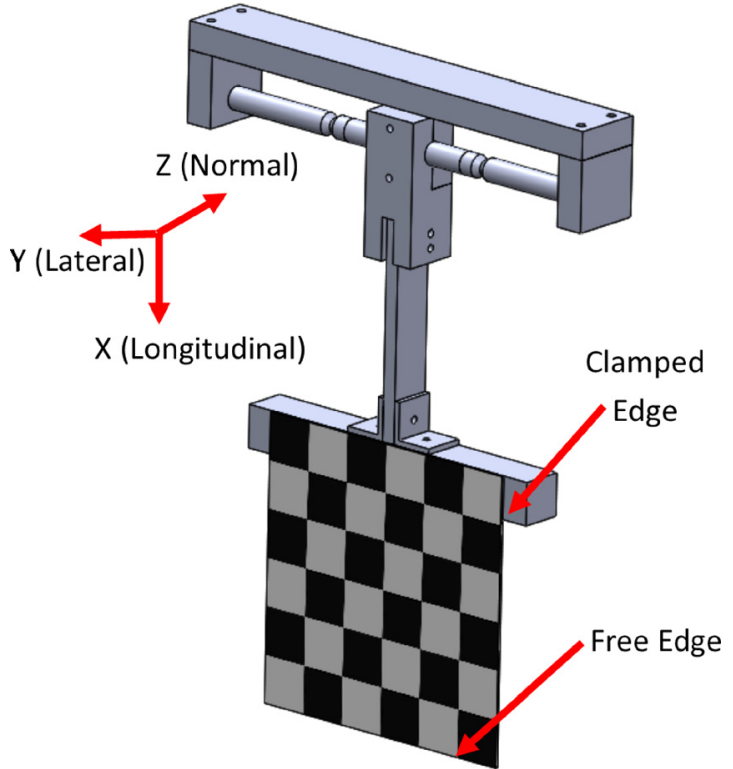

Fig. 1 Schematic of the pendulum rig

Table 1 Parameters for the pendulum case

\begin{tabular}{lcccc}
\hline \hline$\rho_{1}\left(\mathrm{~kg} / \mathrm{m}^{2}\right)$ & $\varphi(\mathrm{N} / \mathrm{m})$ & $\zeta(\mathrm{N} \cdot \mathrm{m})$ & $K_{\mathrm{fsi}}$ & $\Delta t(\mathrm{~s})$ \\
\hline 0.08 & 100 & $1 \times 10^{-4}$ & $2 \times 10^{5}$ & $5 \times 10^{-5}$ \\
\hline \hline
\end{tabular}

$\mathrm{m}^{2}$ [32] to values up to $1.7 \times 10^{-3} \mathrm{~N} \cdot \mathrm{m}$ for a paper of $120 \mathrm{~g} / \mathrm{m}^{2}$ [33]. Eulerian and Lagrangian mesh step sizes are $0.004 \mathrm{~m}$ in a domain of dimensions $(0.5 \mathrm{~m} \times 0.2 \mathrm{~m} \times 0.3 \mathrm{~m})$. The flag is centrally positioned within the domain. The FSI constant $K_{\mathrm{fsi}}$ used for the coupled fluid flag interaction is set at $2 \times 10^{5}$ which is of the same order as reported in Refs. [17] and [18]. It is interesting to note that a one order of magnitude increase in the penalization constant was previously shown to have a non-negligible impact on both the magnitude and the period of oscillations in the case of a clamped rag in crossflow [21]. This classical oscillating flag problem is highly dynamic in the sense that oscillations are sustained by strong feedback between fluid and structure. No such interaction exists in the present case where the flag is driven by the free pendulum oscillations in a quiescent flow. The primary effect of the FSI forces is to dampen the oscillations. In the follow-on cases, the rag motion is determined primarily by the fluid that is with little or no interference with solid boundaries and without a clamping constraint the FSI feedback can again be assumed limited. In both cases, the penalization constant $K_{\mathrm{fsi}}=2 \times 10^{5}$ was chosen to ensure that the immersed structure responded quickly to changes in the flow. Sensitivity tests will be discussed in Sec. 3.2. For the setup of the numerical boundary condition of the flag, the position of the top edge attached to the rigid oscillating object is recorded experimentally and imposed at the clamped edge of the numerically modeled flag.

Digital image correlation method is used to analyze the experimental optical images. The system and analysis rely on stereoscopic imaging of the structure to derive three-dimensional strains and displacements $[34,35]$. The two imaging sensors are located at specific distance with two different orientation angles so that the target flag is within the field of view with sufficient pixelation to capture the displacement at any point on the flag surface. The calculation of each point is obtained with the knowledge of two parameter sets: intrinsic parameters (principal point, focal length, and distortion parameters) and extrinsic parameters (rotation matrix and translation vector which are determined from the position of the two cameras with respect to each other). The DIC system employed for the optical measurement is Q-400 with GigE_Camera 2Mpx provided by Dantec Dynamics GmbH, Ulm, Germany. The lens on each camera is $16 \mathrm{~mm}$. A stochastic speckle pattern is printed on the A4 sheets used in the experiments in order to allow for good quality gray value digital images to be used in the calculation of the displacement. Prior to performing the oscillation tests, the DIC system setup is calibrated and tested for a simple controlled translational and rotational motion and acceptable results were found. For all the experimental tests, an accurate correlation is considered when the residuum is below 0.2 pixels. The captured images are correlated against an initial position with the flag at its vertical equilibrium position. The images were recorded during the test at a frequency of $50 \mathrm{~Hz}$. During the postprocessing stage, the evaluation of the captured images is performed using a facet size of 17 pixels with grid spacing of 14 pixels.

The position of the center point at the free edge is tracked numerically and compared to computational simulations in Figs. 2 and 3 for the normal and the longitudinal directions, respectively. The asymmetrical nature of the oscillations can be explained by the fact that the flag is off-set by a distance of $1 \mathrm{~cm}$ from the vertical equilibrium plane passing through the axis of rotation (see Fig. 1). A maximum error of $7 \%$ and $9 \%$ is observed but this is confined to the peak of the oscillations. Both sets of results clearly

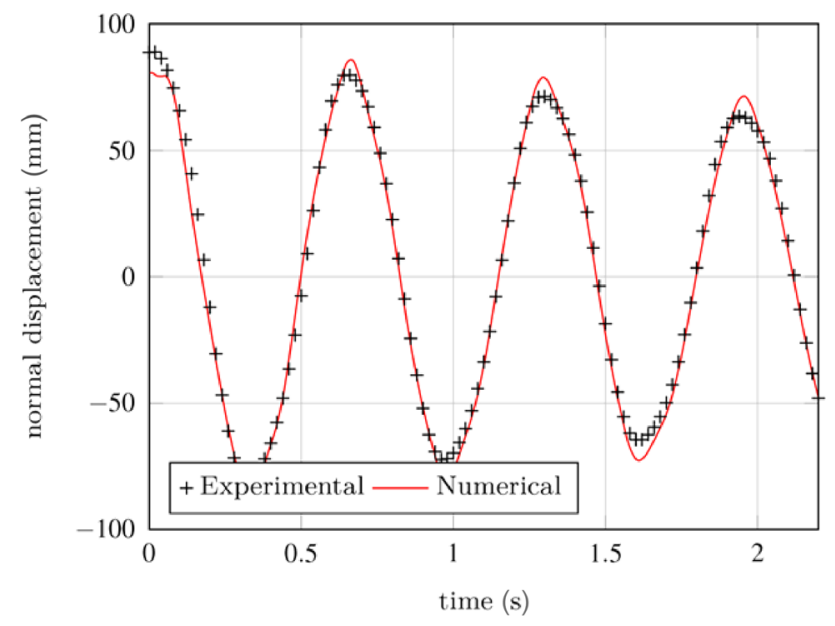

Fig. 2 Time history of the free edge center point displacement in the normal direction

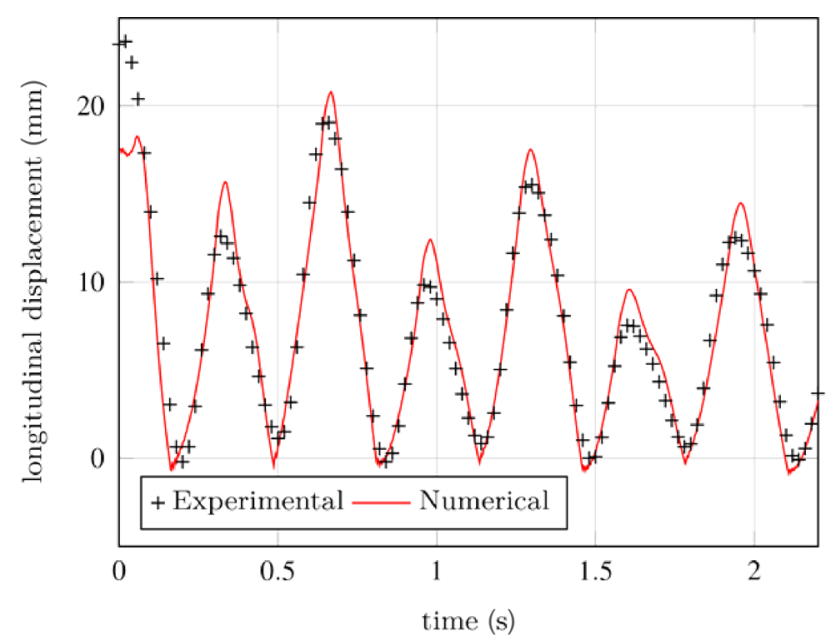

Fig. 3 Time history of the free edge center point displacement in the longitudinal direction 

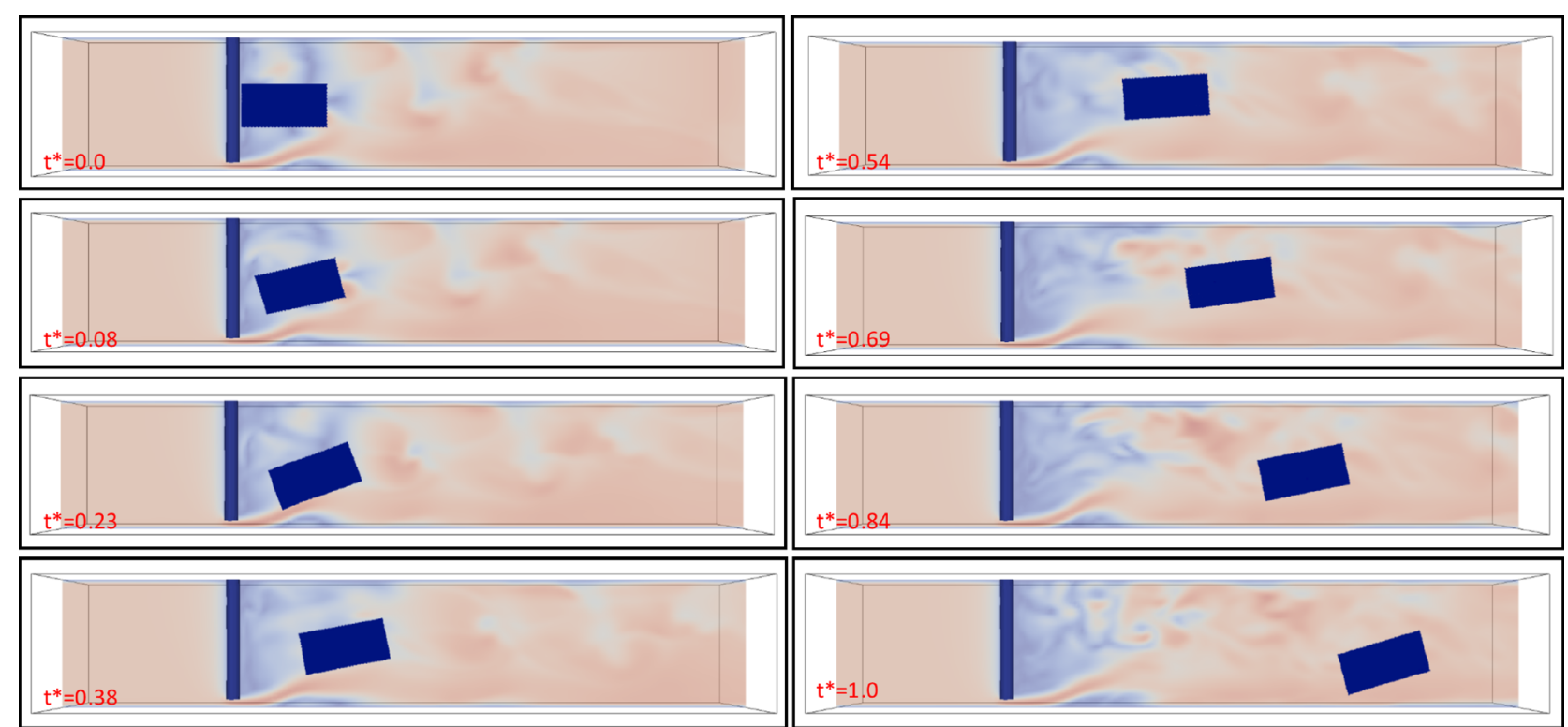

Fig. 4 Snapshots of the rag superimposed on contour plot of flow velocity magnitude from numerical simulations

confirm the effect of damping on the oscillations with a gradual decrease in the amplitudes, and, in general, the FSI solver is shown to provide numerical results that are in close agreement with experimental results both in terms of phase, period, and amplitude of oscillations.

3.2 Free Rag Motion. The aim of this section is to confirm the suitability of the diffuse penalty based IBM to simulate the transport of a free flowing rag. This is achieved by studying the motion and dynamics of a rag in a nonuniform channel flow. Physically, the coupling between the fluid and free flexible objects is affected by both the shear and pressure stresses based on the direction of the fluid flow relative to the surface of the deformable object. When the rag orientation is parallel to the fluid flow, shear stress is the predominant driving force. For more complicated behaviors characterized by arbitrary deformations of the rag, the coupling is affected by both the pressure and shear forces. Numerically, the coupling between the flexible object and the fluid in the FSI solver is represented by an artificial force.

This type of IBM coupling enforces the no-slip and nopenetration conditions at the immersed surface. This is achieved by tuning $K_{\mathrm{fsi}}$ constant to accelerate the fluid in response to structural deformations. Because the source term is applied to the full fluid cell, the no-slip condition does not necessarily need to be strictly enforced in particular when the structure thickness is much smaller than the cell characteristic size. A momentum source defined in terms of the shear stress instead of an arbitrary constant would better capture the underlying physics. On the other hand, the flow should respond very rapidly to the no-penetration condition so that a large $K_{\mathrm{fsi}}$ constant is justified for this condition. As a result, it is expected that the model will not capture correct dynamic responses when the predominant force acting on the immersed surface is due to shear stresses. This, however, would only occur when the slender structure is broadly aligned with streamlines, a condition which is unlikely to prevail in flow with significant mixing and significant dynamic response of the structure.

The test case covered in this section considers the transport of a quasi-neutrally buoyant rag (with $\rho_{s}-\rho_{f} c<0.01 \mathrm{~kg} / \mathrm{m}^{2}$ and a solid density $\rho_{s}=0.08 \mathrm{~kg} / \mathrm{m}^{2}$ ) released in the wake of a cylindrical pole with flow confined in a water tunnel with a square cross section of dimensions $L \times H \times w=0.8 \mathrm{~m} \times 0.15 \mathrm{~m} \times 0.15 \mathrm{~m}$. The numerical domain extends $0.2 \mathrm{~m}$ upstream of the pole axis and $0.6 \mathrm{~m}$ downstream. The Reynolds number studied here is

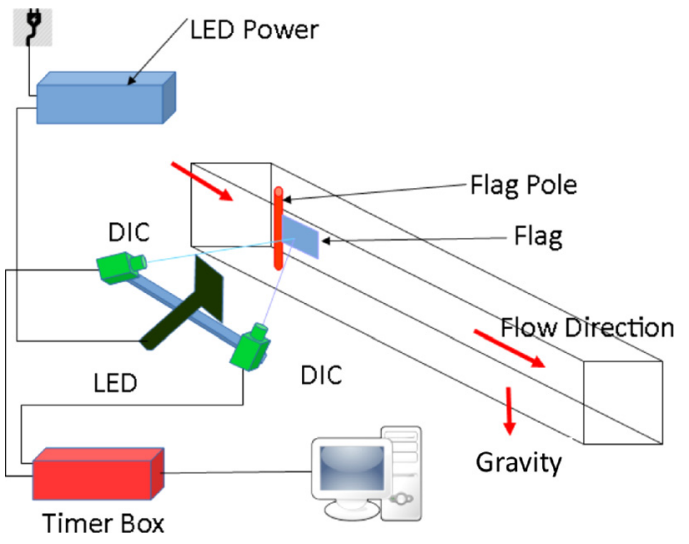

Fig. 5 Schematic diagram of the water tunnel and DIC measurement system

$\operatorname{Re}=0.69 \times 10^{5}$. The blockage due to the pole creates a stream-jet between the lower wall boundary of the tunnel and the pole itself, as illustrated by the numerical results in Fig. 4. The formation of this jet enhances the mixing in the channel and promotes the rotation and deformation of the rag.

The DIC method is used to capture the three-dimensional displacement as shown in Fig. 5. The images were recorded during the test at a frequency of $50 \mathrm{~Hz}$. The rag is positioned so that it is initially parallel to the $x z$ plane behind the flag pole, which has a diameter of $0.016 \mathrm{~m}$ and a height of $0.14 \mathrm{~m}$ and which is located at $0.1 \mathrm{~m}$ away from the inlet boundary. Its initial shape is rectangular with dimensions $0.1 \mathrm{~m} \times 0.05 \mathrm{~m}$ and aligned with the mean flow direction.

For the simulations, both Eulerian and Lagrangian domains are discretized using uniform structured grids with size $\Delta x=0.004 \mathrm{~m}$. The density and the kinematic viscosity of the fluid domain (water) were taken as $1000 \mathrm{~kg} / \mathrm{m}^{3}$ and $1 \times 10^{-6} \mathrm{~m}^{2} / \mathrm{s}$, respectively. The Dirichlet boundary condition is considered at the inflow boundary with a nonzero velocity applied only in the $x$-direction ( $u_{x}=U, u_{y}=0$, where $U$ is the area average streamwise velocity). A constant pressure boundary condition is applied at the outlet and a no-slip wall boundary condition is applied at the top and bottom sides of the numerical domain. The rag free boundary condition is applied at all the sides of the flexible rag so that it moves 
Table 2 Parameters for the free rag motion case

\begin{tabular}{lcccc}
\hline \hline$\rho_{1}\left(\mathrm{~kg} / \mathrm{m}^{2}\right)$ & $\varphi(\mathrm{N} / \mathrm{m})$ & $\zeta(\mathrm{N} \cdot \mathrm{m})$ & $K_{\mathrm{fsi}}$ & $\Delta t(\mathrm{~s})$ \\
\hline$(0.03,0.08)$ & 200 & $1 \times 10^{-4}$ & $2.5 \times 10^{5}$ & $4 \times 10^{-5}$ \\
\hline \hline
\end{tabular}

freely under the effect of the fluid flow. The properties of the rag materials are based on properties of paper and are provided in Table 2 along with the flow characteristics. Numerical tests were performed to determine $K_{\mathrm{fsi}}$ constant over a range varying from $10^{4}$ to $10^{6}$. This involved testing its impact on the terminal velocity and acceleration of a quasi-neutrally buoyant rag placed parallel to a uniform rectilinear flow. The sensitivity analysis showed that with a $K_{\text {fsi }}$ greater than $1 \times 10^{5}$, changes to the acceleration measured as the time to reach the terminal velocity remained within $0.5 \%$ of the reference time. $K_{\mathrm{fsi}}$ was set at $2.5 \times 10^{5}$ for the present case. This large value can be expected to overpredict the acceleration in a flow driven predominantly by shear stress which may be the case at the initial stage of release but not anymore once the rag starts deforming and rotating.

A precursor simulation without the rag but including the cylindrical pole was run to determine the initial pressure and velocity conditions before placing the rag in the flow domain. The initial rag deformation and the dynamic characteristics of its motion (acceleration) were not reproduced in the numerical simulations. As a result, these cannot be expected to capture the exact same dynamic rag response, and the comparison between experimental and numerical results is only intended to assess the time averaged trends while also providing some qualitative descriptions of the rag behavior. A more detailed experimental investigation relying on simultaneous DIC and particle image velocimetry measurements to allow for a more precise comparison of initial conditions is planned as part of follow-on research. The time sequence depicting the simulated and measured motions of the rag after its release is shown in Figs. 4 and 6, respectively. One notable difference between the two results is the initial position of the rag. A delayed release at the lower edge of the rag in the experiments means that it is initially tilted downward due to gravity. This can be expected to lead to stronger interaction with the jet formed at the lower end of the pole.

The experimental and numerical rag mean streamwise and vertical velocities and positions are compared in Figs. 7 and 8 , where $t^{*}=t / U, x^{*}=x / L, y^{*}=y / L$, and $u^{*}=u / U .(x, u)$ and $(y, v)$ are the streamwise and vertical positions and velocities of the centroid of the rag's upstream edge, and $t^{*}=0$ marks the time of release when the upstream rag edge fully detached from the pole.

A comparison between experimental observations and numerical predictions confirms that several key response characteristics are in good agreement. First, the rag is shown to rotate counterclockwise as its tip interacts with the jet formed at the lower end of the flagpole after release. The initial dipping of the flag observed experimentally is due to a delayed release at its lower end which is not modeled numerically. Following release, the rag does not remain in the plane of symmetry of the tunnel and experiences some bending so that the flow streamlines do not remain parallel to rag surface. As these deformations develop, the numerical and experimental transport velocities are shown to converge. These results do indeed suggest that acceleration is overpredicted in shear-dominated flow but that as soon as the rag deforms a better agreement can be expected. For example, $u^{*}$ approaches 1 at $t^{*}=0.69$. Further downstream, Figs. 4 and 6 show that the rag
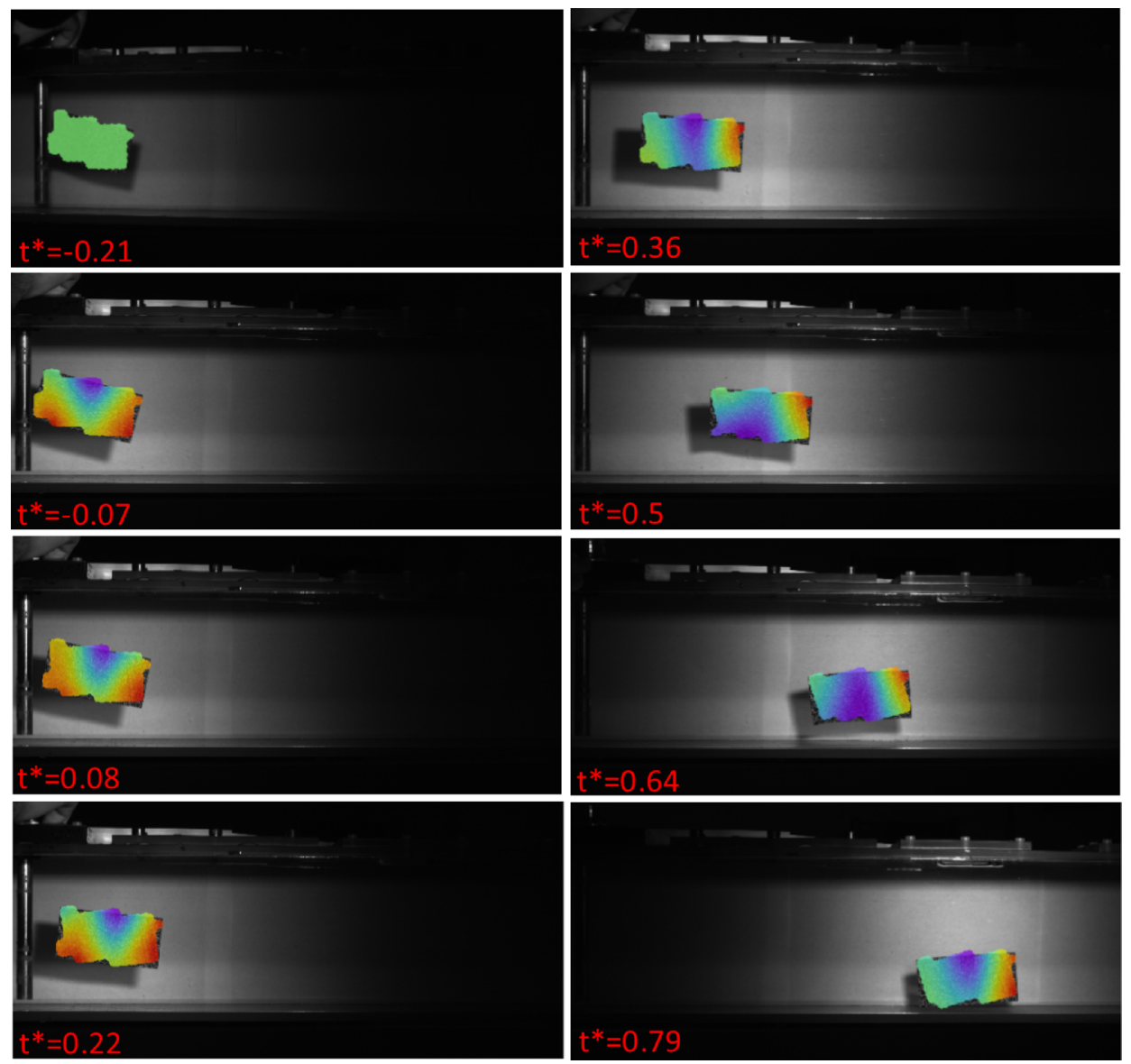

Fig. 6 Transported rag shown with contour plot of transverse displacement obtained by DIC (experimental tests). The flagpole is visible on the left. The nondimension time $t^{\star}$ is shown on each frame. 

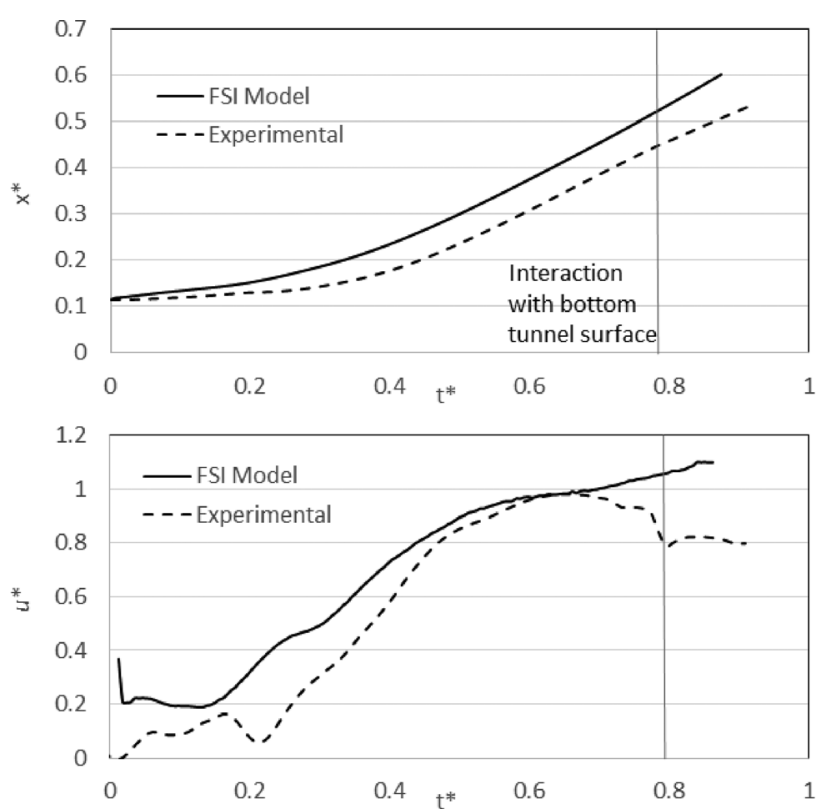

Fig. 7 Comparison between the experimental and numerical motion of the centroid of the rag's upstream edge: displacements of a Lagrangian solid point in the streamwise direction $x$ (top) and streamwise velocity component of the corresponding point (bottom)
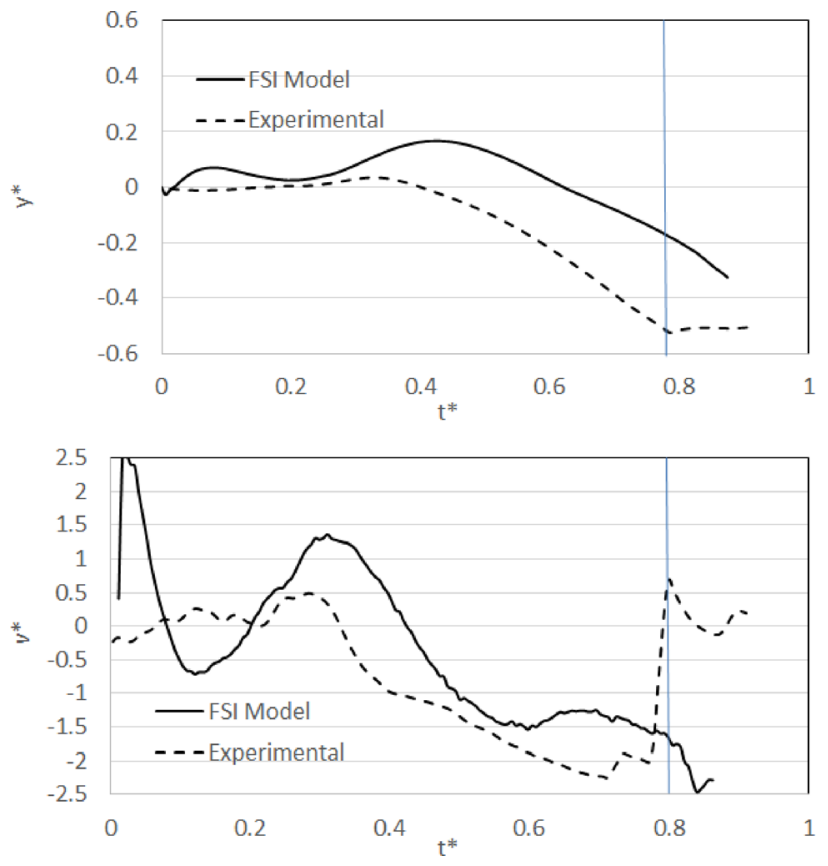

Fig. 8 Comparison between the experimental and numerical motion of the centroid of the rag's upstream edge: displacements of a Lagrangian solid point in the vertical direction $y$ (top) and vertical velocity component of the corresponding point (bottom)

gradually sinks as a result of gravity. This, however, can be seen to occur more rapidly in the experiments. As a result, the rag is seen to collide with the bottom surface of the tunnel at approximately $t^{*}=0.8$ which then translates in a significant drop off in streamwise velocity. These results suggest that although realistic behavior is reproduced by the model, differences can be expected and are most likely due to the numerical coupling approach adopted which are compounded here by fluctuations in the mean streamwise flow velocity produced in the tunnel (maximum fluctuation of $5 \%$ ).

\section{Centrifugal Pumps Simulations}

In this section, practical engineering applications dealing with centrifugal waste water pumps are considered. The sharp IBM from Ref. [15] is first assessed in terms of its ability to model rigid rotation under turbulent flow conditions. Two centrifugal pumps are studied: (i) a single-blade impeller pump and (ii) a two-blade impeller pump (see Fig. 9). First, the hydraulic performance of the pumps is compared to experimental data. Second, the FSI solver is used for the study of rag motion inside the single-blade centrifugal pump under two different flow rates. The objective is here to assess the stability of the solver for cases when the rag experiences large deformations. At the same time, we aim to understand the mechanism of the rag motion through the pump to help identify the key locations where clogging is most likely to occur, and in doing so, support the design optimizations which account for the rag behavior.

4.1 Single-Phase Flow Simulations. The two pumps considered have similar specific speeds with $n_{s}=56.4$ and $n_{s}=50.6$ for the single-blade impeller and the two-blade impeller, respectively. The specific speed is defined as $n_{s}=N \sqrt{Q} /(H)^{0.75}$, where $N$ is the rotational speed (in rpm) and $Q$ and $H$ are, respectively, the flow rate and the hydraulic head delivered by the pump at the best efficiency point (BEP). The mesh size was determined by a compromise between accuracy and computational cost. For both pumps, the ratio of cell size to pump diameter is of the same order, with $1 / 100$ for the single-blade impeller pump and $1 / 75$ for the two-blade impeller pump (see Ref. [36] for details of the mesh sensitivity analysis). This leads to cell sizes of $0.002 \mathrm{~m}$ and 0.005 $\mathrm{m}$, respectively.

Performance studies were performed on both pumps. Five different flow rates $Q$ were considered for single rotational speeds $\omega=150.8 \mathrm{rad} / \mathrm{s}$ and $\omega=77.45 \mathrm{rad} / \mathrm{s}$ for the single-blade impeller and the two-blade impeller, respectively. The density and the kinematic viscosity of the fluid (water) are taken as $1000 \mathrm{~kg} / \mathrm{m}^{3}$ and $1 \times 10^{-6} \mathrm{~m}^{2} / \mathrm{s}$. The hydraulic head and torque results obtained experimentally are compared with the numerical head and torque predicted with the sharp IBM in Figs. 10 and 11 for the two pumps. The IBM is providing head estimates which are in very good agreement with the experimental data around the BEP for the single-blade impeller pump and over the whole range of flow rates for the two-blade impeller pump. For the single-blade impeller pump, the underestimation of the head increases with the flow rate as large flow separation develops over the lower part of the impeller leading edge. Predictions in this case can be expected to be strongly influenced by accurate modeling of the boundary layers and would require much finer wall adjacent mesh. Computations performed with standard body-fitted mesh relying on generalized grid interface (GGI) method have been shown to perform similarly for this pump at equivalent mesh resolution [37]. The torque predictions are, however, less accurate for both pumps and would suggest also that a finer mesh is required. For the two-blade impeller pump, the difference in the torque is around $16 \%$ at low flow rate and increases to $33 \%$ at $Q / Q_{\mathrm{BEP}}=1.6$. Body-fitted simulations have been shown also to underestimate the torque acting on the impeller, with similar discrepancies as for the IBM. Given the good head predictions obtained at low flow rates, and around the BEP, it can be argued that the sharp IBM from Ref. [15] and the present computation model for the single-blade impeller pump are good candidates for FSI simulations of flexible slender structures.

4.2 Rag Transport in the Single-Blade Impeller Pump. Two different flow rates are considered here, $Q=Q_{\mathrm{BEP}}$ and $Q=0.36 Q_{\mathrm{BEP}}$, to characterize the rag transport in the single-blade 


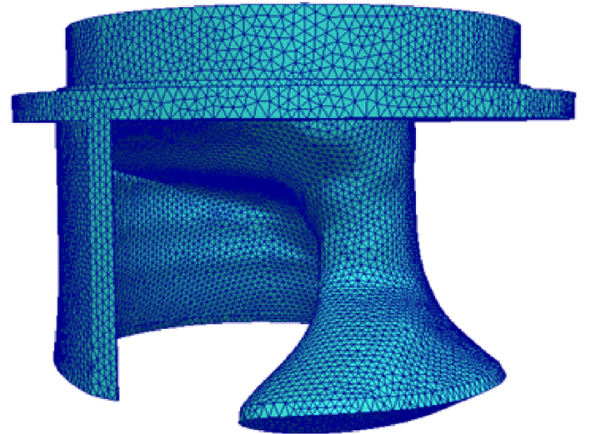

Fig. 9 Surface meshes for IBM model: (left) single-blade impeller and (right) two-blade impeller

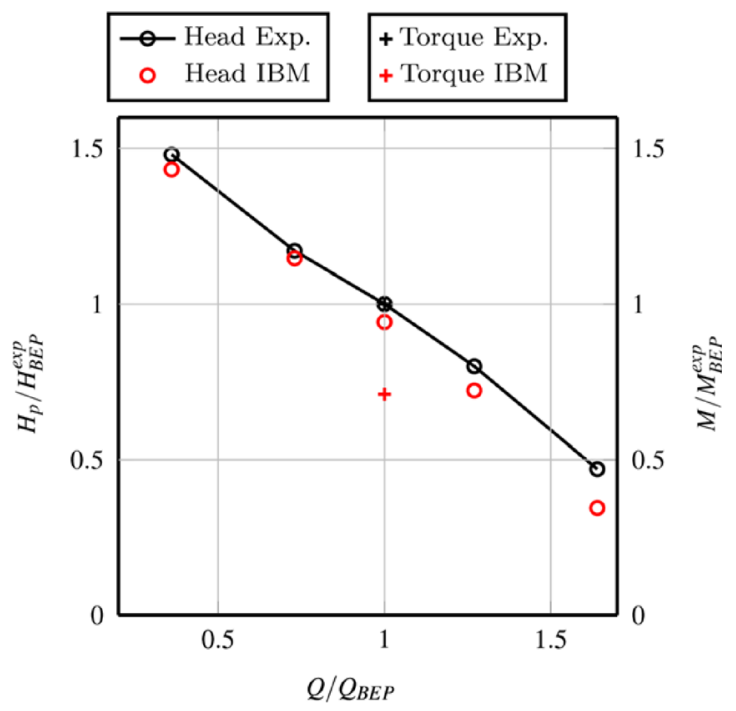

Fig. 10 Head and torque versus flow rates for the single-blade impeller pump. All data are normalized by the experimental head and torque at the BEP. Experimental data are provided by Sulzer.

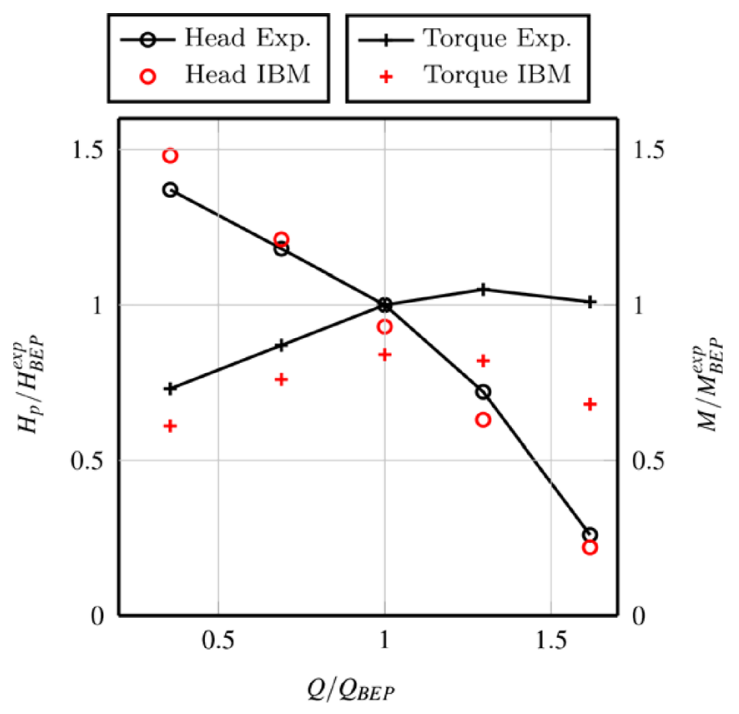

Fig. 11 Head and torque versus flow rates for the two-blade impeller pump. All data are normalized by the experimental head and torque at the BEP. Experimental data are provided by Sulzer.

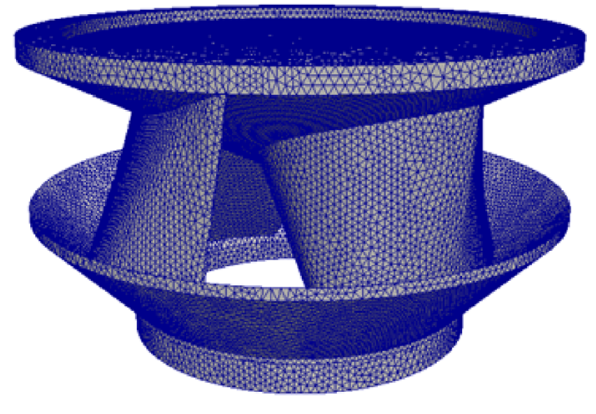

impeller pump. The rag is initially positioned vertically at the bottom of the volute inside the inlet sump and upstream of the singleblade impeller. Two different sizes for the rag are considered: $10 \mathrm{~cm} \times 10 \mathrm{~cm}$ and $15 \mathrm{~cm} \times 5 \mathrm{~cm}$. The step size of $0.004 \mathrm{~m}$ for the resolution of the Lagrangian domain was determined based on the results from the previous benchmark cases. The distance threshold in the collision model introduced in Sec. 2.5 is fixed to 2 cells Eulerian width, which is $0.004 \mathrm{~m}$. The free boundary condition is applied at all the sides of the flexible rag so that it moves freely under the effect of the fluid flow. The Dirichlet boundary condition is considered at the inflow satisfying the imposed volume flow rate, while a constant pressure boundary condition and a noslip wall boundary condition are applied at the outflow boundary and at all walls, respectively. The density difference in the solid solver is selected as $\rho_{1}=0.03 \mathrm{~kg} / \mathrm{m}^{2}$. The bending rigidity is selected as $\zeta=1 \times 10^{-8} \mathrm{~N} \cdot \mathrm{m}$. Very small values for the bending rigidity are used to allow for the large rag deformations observed experimentally in tunnel experiments with soaked paper interacting with turbulent flow past a cylindrical obstacle. A tension coefficient $\varphi=10 \mathrm{~N} / \mathrm{m}$ is used so that the rag does not experience any significant stretching. This coefficient has been shown to maintain the maximum stretching below $3 \%$, while larger values lead to numerical instabilities in the solid solver. The gravitational acceleration $\left(g=9.81 \mathrm{~m} / \mathrm{s}^{2}\right)$ is applied along the vertical direction. The time-step size and IBM constant were selected as $\Delta t=5 \times 10^{-5} \mathrm{~s}$ and $K_{\mathrm{fsi}}=1 \times 10^{5}$, respectively.

Figure 12 shows the time sequence for the rag during its motion inside the pump at a flow rate $Q_{\mathrm{BEP}}$ equivalent to the $\mathrm{BEP}$, for the long rag $(15 \mathrm{~cm} \times 5 \mathrm{~cm})$. The low pressure in the eye of the impeller forces the rag to travel vertically upward along the axis of rotation toward the impeller. It is then pushed outside the impeller region by the action of centrifugal force. The strong mixing inside the pump leads to large rag deformations. As the rag exits the inner region past the leading edge of the impeller, it wraps itself temporarily around the high curvature surface of the impeller leading edge. This tends to occur over a fraction of a rotor revolution period but the wrapping is also shown to increase as the pump flow rate decreases. The current FSI model assumes that a thin liquid film forms and remains between the rag and the impeller wall so that solid-solid friction cannot be directly modeled. It can be expected that as a result the friction is underestimated and determined by viscous liquid shear only. When the rag leaves impeller zone, it travels along the volute in the anticlockwise direction until it leaves the pump through the discharge pipe. The visualization of the rag inside the pump highlights three different locations where the rag might get trapped on a solid surface potentially leading to blockage: (i) the impeller eye, where partial dead zone regions are shown to form, (ii) the leading edge of the impeller, and finally, (iii) the cut water region where the volute meets with the discharge pipe. Figure 12 shows clearly how the rag wraps around the cut water.

The study of the rag at lower flow rate $\left(Q / Q_{\mathrm{BEP}}=0.36\right)$ is shown in Fig. 13. A similar general dynamics trend has been 

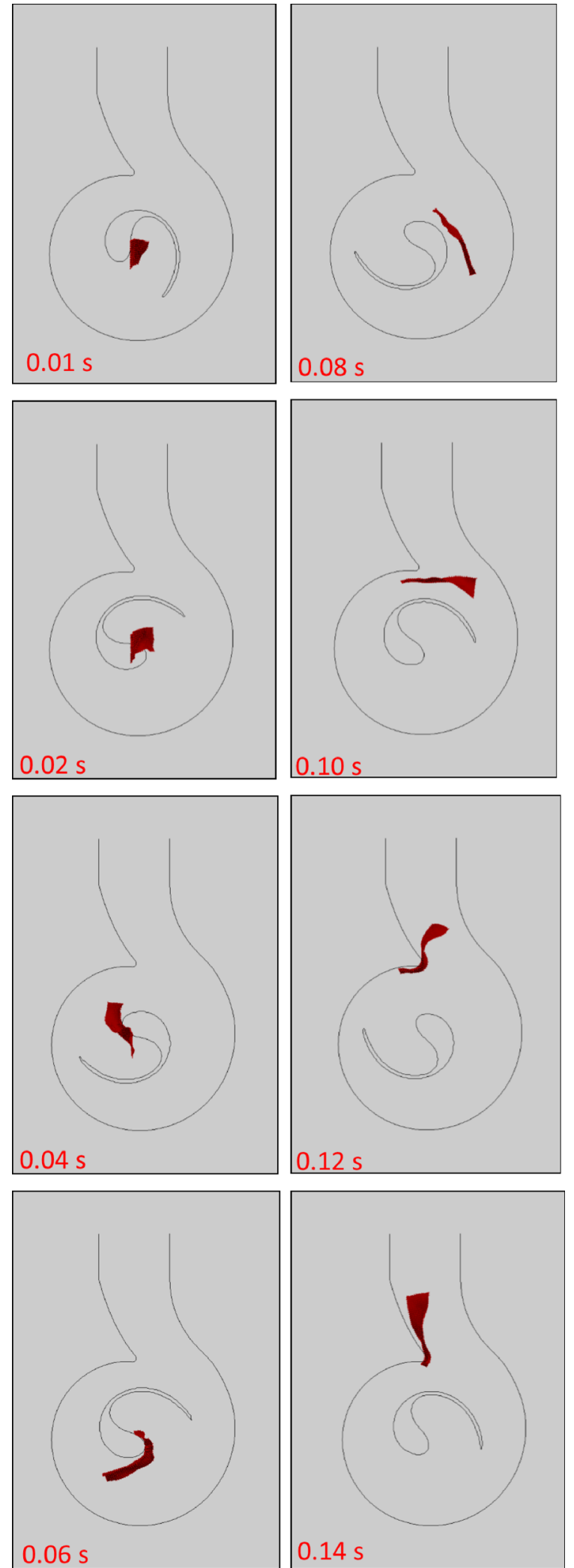

Fig. 12 Visualization of the deformed rag surface at eight successive times after the release for $Q / Q_{\mathrm{BEP}}=1$. The cross section of the pump volute and impeller is shown in gray.

observed in this case compared to the BEP. However, three differences have been observed compared to the previous case. First, the rag wraps around the leading edge at a different height. Second, the rag does not collide against the cut water region
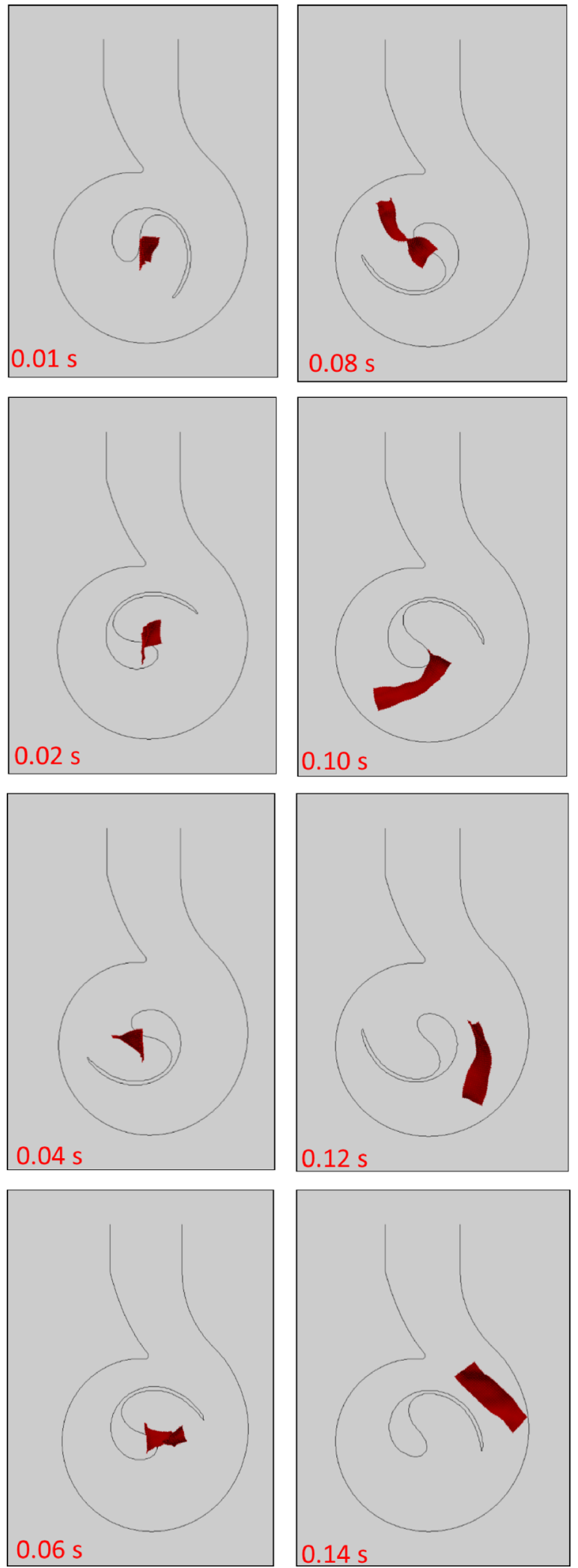

Fig. 13 Visualization of the deformed rag surface at eight successive times after the release for $Q / Q_{\mathrm{BEP}}=0.36$. The cross section of the pump volute and impeller is shown in gray.

indicating the strong sensitivity of the results to the initial and boundary conditions. Finally, there is a significant delay in the time required for the rag to leave the impeller region, and therefore, the pump. This can be inferred to increase the likelihood of 


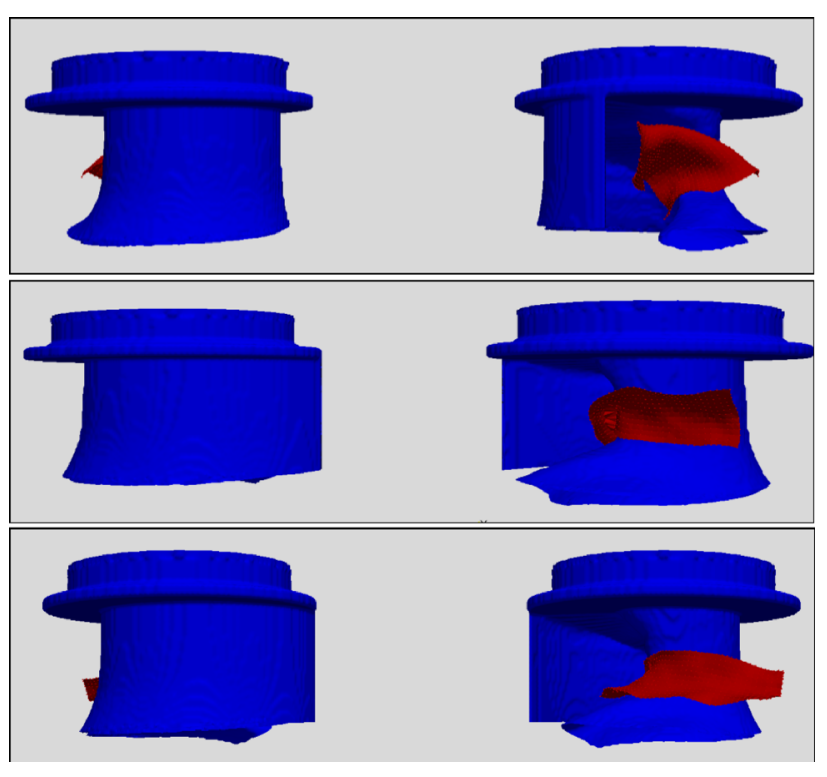

Fig. 14 Visualization of the rag (shown in red) as it wraps itself around the impeller (shown in blue): square $10 \mathrm{~cm} \times 10 \mathrm{~cm}$ rag at $Q / Q_{\mathrm{BEP}}=1$ (top), rectangular $15 \mathrm{~cm} \times 5 \mathrm{~cm}$ rag at $Q / Q_{\mathrm{BEP}}=1$ (middle), and rectangular $15 \mathrm{~cm} \times 5 \mathrm{~cm}$ rag at $Q / Q_{\mathrm{BEP}}=0.36$ (bottom)

the rag being wrapped around the leading edge for a long time allowing for multiple rags to accumulate. At $Q / Q_{\mathrm{BEP}}=0.36$, the rag remains around the leading edge for approximately $50 \%$ of the simulation time (or approximately 2.4 periods of revolution) instead of $30 \%$ at $Q_{\mathrm{BEP}}$ (or 1.5 periods of revolution). Hence, the rag takes only $0.1 \mathrm{~s}$ to reach the cut water region at the BEP compared to $0.14 \mathrm{~s}$ at the lower flow rate. Similar conclusions regarding the probability of clogging were obtained experimentally in Ref. [4]. Inspection of the flow in Ref. [38] suggests that changing the flow rate leads to a change in fluid incident angle relative to the impeller leading edge. Increasing the flow generates area of increased radial velocity and decreased tangential velocity, as observed also in Ref. [39].

Figure 14 shows the position of the rag at the impeller leading edge for three different rags: the results show that wide rags are more likely to find themselves wrap around the whole leading edge. In contrast, long narrow rag wraps around a small region of the leading edge. For the BEP, the rag wraps around the top side of the leading edge close to the hub. In contrast, the wrap moves around the bottom side at smaller flow rates. These observations highlight the strong sensitivity of rag dynamics to the size of the rag and the value of the flow rate, and hence, the importance of a holistic approach to design optimization if clogging dynamics is to be accounted for. It is also worth noting that experimental observations from high-speed camera imaging of rag flow at the inlet sump of the same reference pump confirmed that rags that remain within the pump volute tend to wrap around the leading edge of the impeller. This behavior was observed from visualization tests obtained with high-speed cameras and confirmed following the dismantling of the impeller. Statistical assessment of experimental studies have indicated that the likelihood of blockage is based on several factors which include the rag initial location, the fluid inlet boundary conditions, the geometrical parameters for the pump hydraulic parts, and the rag geometrical and physical properties.

\section{Conclusion}

A FSI solver based on IBMs has been described and validated for multiple interactions between flows from low to high Reynolds numbers, flexible slender structures, and rigid moving bodies. The solver couples a diffuse IBM with a finite difference solution of the motion equation of a two-dimensional elastic solid and a sharp IBM for rigid body motion including a collision model to account for interactions with moving immersed objects. It has been assessed with a range of benchmark cases including a highly dynamic system at different flow regimes. Comparisons with the previously published FSI solvers based on IBMs and new experimental tests presented in this paper indicate that stable, robust, and accurate or physically realistic predictions can be achieved. The solver was then applied to study the transport of flexible structures through a centrifugal pump and describe the processes that may lead to accumulation of rags around impeller. Results clearly highlight the influence of two key parameters which are the rag length and the pump flow rate on the positioning of the rag within the volute and around the impeller providing for the first time some insight in the dynamics of the rag and its sensitivity to flow conditions.

\section{Acknowledgment}

The authors would like to thank the staff of Sulzer pump Wexford for their support in selecting suitable test cases and providing experimental validation data. All numerical simulations were performed on the FIONN cluster of the Science Foundation Ireland/ Higher Education Authority (SFI/HEA) Irish Centre for High-End Computing (ICHEC). ICHEC support through the dceng002b and dceng005b Class B Projects is gratefully acknowledged.

\section{Funding Data}

- Enterprise Ireland (EI) (IP/2014/0359).

- Irish Research Council (IRC) (EBPPG 2013 63).

\section{References}

[1] Gerlach, S., and Thamsen, P. U., 2017, "Cleaning Sequence Counters Clogging: A Quantitative Assessment Under Real Operation Conditions of a Wastewater Pump," ASME Paper No. FEDSM2017-69020.

[2] Jensen, A. L., Gerlach, S., Lykholt-Ustrup, F., Sørensen, H., Rosendahl, L., and Thamsen, P. U., 2017, "Investigation of the Influence of Operating Point on the Shape and Position of Textile Material in the Inlet Pipe to a Dry-Installed Wastewater Pump," ASME Paper No. FEDSM2017-69298.

[3] Pöhler, M., Höchel, K., and Gerlach, S., 2017, "Linking Efficiency to Functional Performance by a Pump Test Standard for Wastewater Pumps," ASME Paper No. AJKFluids2015-33763.

[4] Connolly, R., 2017, "An Experimental and Numerical Investigation Into Flow Phenomena Leading to Wastewater Centrifugal Pump Blockage," ME thesis, Dublin City University, Dublin, Ireland.

[5] Jensen, A. L., Sørensen, H., Rosendahl, L., and Thamsen, P. U., 2018, "Characterisation of Textile Shape and Position Upstream of a Wastewater Pump Under Different Part Load Conditions," Urban Water J., 15(2), pp. 132-137.

[6] Jensen, A. L., Sørensen, H., and Rosendahl, L., 2016, "Towards Simulation of Clogging Effects in Wastewater Pumps: Modelling of Fluid Forces on a Fiber of Bonded Particles Using a Coupled CFD-DEM Approach," International Symposium on Transport Phenomena and Dynamics of Rotating Machinery (ISROMAC 2016), Honolulu, HI, Apr. 10-15, pp. 1-6.

[7] Jensen, A. L., Sørensen, H., Rosendahl, L., Adamsen, P., and Lykholt-Ustrup, F., 2016, "Investigation of Drag Force on Fibres of Bonded Spherical Elements Using a Coupled CFD-DEM Approach," Ninth International Conference on Multiphase Flow (ICMF), Firenze, Italy, May 22-27, Paper No. 1922.

[8] Yao, Z.-F., Yang, Z.-J., and Wang, F.-J., 2016, "Evaluation of Near-Wall Solution Approaches for Large-Eddy Simulations of Flow in a Centrifugal Pump Impeller," Eng. Appl. Comput. Fluid Mech., 10(1), pp. 454-467.

[9] Posa, A., Lippolis, A., and Balaras, E., 2016, "Investigation of Separation Phenomena in a Radial Pump at Reduced Flow Rate by Large-Eddy Simulation," ASME J. Fluids Eng., 138(12), p. 121101.

[10] Pei, J., Yuan, S., Benra, F.-K., and Dohmen, H., 2012, "Numerical Prediction of Unsteady Pressure Field Within the Whole Flow Passage of a Radial SingleBlade Pump," ASME J. Fluids Eng., 134(10), p. 101103.

[11] Pei, J., Dohmen, H., Yuan, S., and Benra, F.-K., 2012, "Investigation of Unsteady Flow-Induced Impeller Oscillations of a Single-Blade Pump Under Off-Design Conditions," J. Fluids Struct., 35, pp. 89-104.

[12] Souza, B. D., and Niven, A., 2008, "Single Blade Impeller Development Through the Use of the Design of Experiments Method in Combination With Numerical Simulation," International Symposium on Transport Phenomena and Dynamics of Rotating Machinery (ISROMAC 2008), Honolulu, HI, Feb. 17-22, pp. 1-8. 
[13] de Souza, B., Niven, A., and McEvoy, R., 2010, "A Numerical Investigation of the Constant-Velocity Volute Design Approach as Applied to the Single Blade Impeller Pump," ASME J. Fluids Eng., 132(6), p. 061103.

[14] Stephen, C., Yuan, S., Pei, J., and Cheng, G. X., 2017, "Numerical Flow Prediction in Inlet Pipe of Vertical Inline Pump," ASME J. Fluids Eng., 140(5), p. 051201.

[15] Specklin, M., and Delauré, Y., 2018, "A Sharp Immersed Boundary Method Based on Penalisation and Its Application to Moving Boundaries and Turbulent Rotating Flows," Eur. J. Mech./B Fluids, 70, pp. 130-147.

[16] Huang, W.-X., Shin, S. J., and Sung, H. J., 2007, "Simulation of Flexible Filaments in a Uniform Flow by the Immersed Boundary Method," J. Comput. Phys., 226(2), pp. 2206-2228.

[17] Huang, W.-X., and Sung, H. J., 2010, "Three-Dimensional Simulation of Flapping Flag in a Uniform Flow," J. Fluid Mech., 653, pp. 301-336.

[18] Huang, W.-X., and Sung, H. J., 2009, "An Immersed Boundary Method for Fluid Flexible Structure Interaction," Comput. Methods Appl. Mech. Eng., 198(33-36), pp. 2650-2661.

[19] Ryu, J., Park, S. G., and Sung, H. J., 2018, "Flapping Dynamics of Inverted Flags in a Side-by-Side Arrangement," Int. J. Heat Fluid Flow, 70, pp. 131-140.

[20] Park, S. G., and Sung, H. J., 2018, "Hydrodynamics of Flexible Fins Propelled in Tandem, Diagonal, Triangular and Diamond Configurations," J. Fluid Mech. 840, pp. 154-189.

[21] Albadawi, A., Marry, S., Breen, B., Connolly, R., and Delauré, Y., 2016, "An IBM-FSI Solver of Flexible Objects in Fluid Flow for Pumps Clogging Applications," Ninth International Conference on Computational Fluid Dynamics (ICCFD9), Istanbul, Turkey, July 11-15, pp. 1-22.

[22] Tian, F.-B., Dai, H., Luo, H., Doyle, J. F., and Rousseau, B., 2014, "FluidStructure Interaction Involving Large Deformations: 3D Simulations and Applications to Biological Systems," J. Comput. Phys., 258, pp. 451-469.

[23] Lee, I., and Choi, H., 2015, "A Discrete-Forcing Immersed Boundary Method for the Fluid-Structure Interaction of an Elastic Slender Body," J. Comput. Phys., 280, pp. 529-546.

[24] de Tullio, M., and Pascazio, G., 2016, "A Moving-Least-Squares Immersed Boundary Method for Simulating the Fluid-Structure Interaction of Elastic Bodies With Arbitrary Thickness," J. Comput. Phys., 325, pp. 201-225. (August),

[25] OpenCFD, 2016, "The Open Source Computational Fluid Dynamics (CFD) Toolbox," ESI Group, Paris, France, http://openfoam.com/
[26] Issa, R., 1986, "Solution of the Implicitly Discretised Fluid Flow Equations by Operator-Splitting," J. Comput. Phys., 62(1), pp. 40-65.

[27] Weller, H., 2012, "Controlling the Computational Modes of the Arbitrarily Structured c Grid," Mon. Weather Rev., 140(10), pp. 3220-3234.

[28] Lysenko, D. A., and Rian, K. R., 2014, "Large-Eddy Simulation of the Flow Over a Cylinder at Reynolds Number $2 \times 10^{4}$," Flow Turbul. Combust., 92(3), pp. 673-698.

[29] Krastev, V., and Bella, G., 2012, “A Zonal Turbulence Modeling Approach for Ice Flow Simulation," SAE Int. J. Engines, 9(3), pp. 1425-1436.

[30] Spalart, P. R., Jou, W.-H., Strelets, M., and A, S. R., 1997, "Comments on the Feasibility of LES for Wings and on a Hybrid RANS/LES Approach," Advances in DNS/LES: Proceedings of the First AFOSR International Conference on DNS/LES, Louisiana Tech University, Ruston, Louisiana, USA, August 4-8, 1997, Greyden Press, Columbus, OH

[31] Spalding, D., 1961, "A Single Formula for the Law of the Wall," ASME J. Appl. Mech., 28(3), pp. 455-458.

[32] Fällström, K. E., Gren, P., and Mattsson, R., 2002, "Determination of Paper Stiffness and Anisotropy From Recorded Bending Waves in Paper Subjected to Tensile Forces," NDT and E Int., 35(7), pp. 465-472.

[33] Virot, E., Amandolese, X., and Hémon, P., 2013, "Fluttering Flags: An Experimental Study of Fluid Forces," J. Fluids Struct., 43, pp. 385-401.

[34] Siebert, T., and Crompton, M. J., 2013, "Application of High Speed Digital Image Correlation for Vibration Mode Shape Analysis," Application of Imaging Techniques to Mechanics of Materials and Structures, Vol. 4, Springer, Berlin, pp. 291-298.

[35] Tabandeh-Khorshid, M., Schultz, B., Rohatgi, P., and Elhajjar, R., 2016, "The Diametrically Loaded Cylinder for the Study of Nanostructured AluminumGraphene and Aluminum-Alumina Nanocomposites Using Digital Image Correlation," Front. Mater., 3, p. 22.

[36] Specklin, M., 2018, "On the Assessment of Immersed Boundary Methods for FluidStructure Interaction Modelling: Application to Waste Water Pumps Design and the Inherent Clogging Issues," Ph.D. thesis, Dublin City University, Dublin, Ireland.

[37] Specklin, M., 2018, "A Versatile Immersed Boundary Method for Pump Design," Sulzer Management Ltd., Winterthur, Switzerland, White Paper, $1 / 2018$.

[38] Gülich, J. F., 2008, Centrifugal Pumps, Springer, Berlin.

[39] Feng, J., Benra, F. K., and Dohmen, H. J., 2009, "Unsteady Flow Visualization at Part-Load Conditions of a Radial Diffuser Pump: By PIV and CFD," J. Visualization, 12(1), pp. 65-72. 